\section{Anaphylaxien nach Arzneimittelgabe}

Unverträglichkeiten gegenüber Arzneimitteln treten häufig auf. Abzugrenzen sind dosisabhängige Nebenwirkungen von immunologisch vermittelten Unverträglichkeiten. Eine Anaphylaxie ist eine akut einsetzende, systemische, mastzellabhängige Reaktion, bei der durch Degranulation von Mastzellen Symptome an der Haut im Sinne einer Urtikaria oder von Angioödemen, aber auch respiratorische, kardiovaskuläre und gastrointestinale Symptome auftreten können. Wenn mehr als zwei Organsysteme betroffen sind, spricht man von einer Anaphylaxie.

Anaphylaktische Reaktionen können entweder IgE-vermittelt durch Medikamente ausgelöst werden, oder es kommt über Aktivierung eines G-Protein-gekoppelten Rezeptors zu einer direkten Mastzelldegranulation. Medikamente, die sehr häufig solche Reaktionen auslösen, sind Betalactam-Antibiotika und nicht-steroidale
Antiphlogistika. Während die BetalactamAntibiotika wie Penicilline und Cephalosporine häufig IgE-vermittelte Reaktionen auslösen [1], können beispielsweise Chinolone über einen nicht-lgE-abhängigen Mechanismus Mastzellen aktivieren. Unter den Analgetika sind die häufigsten Auslöser Acetylsalicylsäure, Diclofenac, Ibuprofen, aber auch beispielsweise Paracetamol [2]. Arzneimittelinduzierte Anaphylaxien treten am häufigsten ab der 5. Lebensdekade auf und stellen in höheren Altersgruppen den häufigsten Auslöser schwerer anaphylaktischer Reaktionen dar. Nur mittels hinreichender Identifizierung des Auslösers ist es möglich, Patienten zu beraten und Ausweichpräparate zu identifizieren, um eine erneute Exposition mit möglicher Todesfolge zu vermeiden.

Interessenkonflikt

Die Autorin gibt an, dass kein Interessenkonflikt besteht.
Autorin

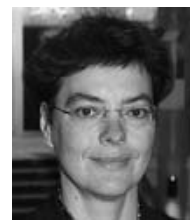

\section{Margitta Worm}

Prof. Dr. med., Allergologie und Immunologie, Klinik für Dermatologie, Venerologie und Allergologie, Charité Campus Mitte, Universitätsmedizin Berlin

\section{Korrespondenzadresse}

Prof. Dr. med. Margitta Worm Allergologie und Immunologie Klinik für Dermatologie, Venerologie und Allergologie

Charité Campus Mitte

Universitätsmedizin Berlin (CCM)

10117 Berlin

Deutschland

margitta.worm@charite.de

Literatur

[1] Worm M, Moneret-Vautrin A, Scherer K et al. First European data from the network of severe allergic reactions (NORA). Allergy 2014; 69: 1397-1404

[2] Worm M. Anaphylaxie Wie richtig handeln? Perspektiven der Pneumologie und Allergologie 1/2018 | Deutsches Ärzteblatt 